\title{
Mini Review
}

\section{Rheumatic heart disease in the modern era: recent developments and current challenges}

\author{
Matheus Tozatto Baptista Coelho Leal ${ }^{[1]}$, Livia Silva Araújo Passos ${ }^{[1],[2],}$ \\ Felipe Vieira Guarçoni[ ${ }^{[1]}$, João Marcelo de Souza Aguiar ${ }^{[1]}$, Rafael Benjamim Rosa da Silva ${ }^{[1]}$, \\ Thiago Mendonça Nunes de Paula ${ }^{[1]}$, Rafael Figueiredo dos Santos ${ }^{[1]}$, \\ Maria Cecília Landim Nassif ${ }^{11]}$, Nayana F. A. Gomes ${ }^{[1]}$, Timothy C. Tan ${ }^{[3]}$ \\ and Maria Carmo Pereira Nunes ${ }^{[1]}$
}

\begin{abstract}
[1]. Faculdade de Medicina da Universidade Federal de Minas Gerais, Belo Horizonte, MG, Brasil. [2]. The Center for Excellence in Vascular Biology, Brigham and Women's Hospital, Harvard Medical School, Boston, MA, USA.

[3]. Department of Cardiology, Blacktown Hospital, University of Western Sydney, NSW, Australia.
\end{abstract}

\begin{abstract}
Rheumatic heart disease (RHD) remains a major cause of preventable death and disability in children and young adults. Despite significant advances in medical technology and increased understanding of disease mechanisms, RHD continues to be a serious public health problem throughout the world, especially in low- and middle-income countries. Echocardiographic screening has played a key role in improving the accuracy of diagnosing RHD and has highlighted the disease burden. Most affected patients present with severe valve disease and limited access to life-saving cardiac surgery or percutaneous valve intervention, contributing to increased mortality and other complications. Although understanding of disease pathogenesis has advanced in recent years, key questions remain to be addressed. Preventing or providing early treatment for streptococcal infections is the most important step in reducing the burden of this disease.
\end{abstract}

Keywords: Rheumatic heart disease. Echocardiographic screening. Pathogenesis. Valve disease.

\section{INTRODUCTION: OVERVIEW OF RHEUMATIC HEART DISEASE}

Rheumatic heart disease (RHD) is a chronic valvular disease caused by heart valve damage from severe or repetitive episodes of acute rheumatic fever (ARF) ${ }^{1-4}$. ARF results from an autoimmune response to infection caused by Group A Streptococcus (GAS) in genetically susceptible individuals ${ }^{5}$. As the acute illness resolves, the valvular lesions tend to persist and progress over time, leading to chronic RHD, which is the major cause of morbidity and mortality from $A R F^{1,5}$. These key definitions are presented in Table $\mathbf{1}$.

RHD remains a major public health concern, especially in low- and middle-income countries, where it is the leading

Corresponding author: Prof. Maria Carmo Pereira Nunes.

e-mail: mcarmo@waymail.com.br

ORCID: 0000-0001-8801-1828

Received 22 January 2019

Accepted 5 Febrary 2019 cause of cardiovascular death and disability in children and young adults ${ }^{6-8}$. There are an estimated 33.4 million individuals currently living with $\mathrm{RHD}^{6}$. The disease was responsible for 305,000 deaths in 2015 , and more than one million premature deaths annually ${ }^{6}$.

Awareness of RHD has increased recently, owing to an improved understanding of the true burden of disease resulting from echocardiography-based screening studies in countries in which the condition remains endemic ${ }^{9,10}$. In addition, there has been a sustained increase in focus on RHD among researchers in RHD-endemic countries ${ }^{1,11,12}$. In 2015, RHD Action, a civil society movement, was launched to increase support and awareness for the early diagnosis and treatment of RHD ${ }^{9}$. More recently, the World Health Assembly adopted a resolution to reinvigorate global and national interest in preventative and management strategies for ARF and RHD ${ }^{13,14}$.

The strong link between RHD and low- and middle-income countries highlights a series of factors related to its evolution, from the point of diagnosis to advanced disease stages. Other key issues include failure to receive adequate secondary prophylaxis 
TABLE 1: Key definitions.

Acute rheumatic fever

An acute illness caused by an autoimmune response to infection with Group A Streptococcus, leading to a range of possible symptoms and signs affecting any or all of heart, joints, brain, skin, and subcutaneous tissues. Acute rheumatic fever is diagnosed according to the Revised Jones Criteria and has a tendency to recur with subsequent Group A streptococcal infections.

Rheumatic carditis

Active inflammation of the heart tissues, most importantly the mitral and/or the aortic valves, caused by acute rheumatic fever. Rheumatic carditis can lead to chronic damage that remains after the acute inflammatory episode has resolved.

\section{Rheumatic heart disease}

Persistent damage to heart valves resulting in mitral and/or aortic regurgitation, or stenosis in long-standing cases, that remains as a result of acute rheumatic fever with rheumatic carditis. Complications of rheumatic heart disease include heart failure, embolic stroke, endocarditis, and atrial fibrillation.

Source: Carapetis, JR. el at. Nat Rev Dis Primers. 2016 14;2:15084.

and inadequate monitoring of anticoagulation, which may increase the incidence of complications such as stroke and bleeding ${ }^{12,15}$. However, epidemiological elimination of the disease extends far beyond ambulatory or hospital measures, and will require political-economic-administrative behavioral change, at the municipal, state, and federal level, with the capability to articulate and consolidate three key fundamental parameters: prevention, health policies and advanced care ${ }^{12}$.

Although the incidence of ARF is decreasing worldwide, the rise in RHD prevalence is mainly occurring as a result of increased survival of patients with valve disease and the availability of echocardiography to improve the diagnosis of $\mathrm{RHD}^{3}$. Major advances in medical and surgical treatments for RHD have led to increased survival, which may also contribute to an increased prevalence of $\mathrm{RHD}^{3,6}$. Although valve replacement provides good early results, the patients usually have difficulty in accessing medical care, with a high rate of prosthesis-related complications ${ }^{16}$. Unfortunately, many patients present with valve dysfunction too advanced to benefit from cardiac surgery, and the mortality in these patients remains high ${ }^{15,17,18}$.

The objective of this review is to provide an update on RHD, highlighting epidemiological concepts, improvement in diagnosis using echocardiographic screening, current understanding of the pathogenesis of valvular structural abnormalities, and, finally, effective preventive strategies currently employed in developing countries.

\section{EPIDEMIOLOGY: GLOBAL BURDEN OF THE DISEASE}

The incidence of acute rheumatic fever (ARF) and of its long-term sequela, rheumatic heart disease (RHD), is low in high-income countries, mainly owing to improvements in the socioeconomic condition of the population and efficient infection control with benzathine penicillin $\mathrm{G}(\mathrm{BPG})^{6,10}$. However, in a few developed countries such as Australia and New Zealand, RHD remains a relevant issue, particularly in indigenous populations, such as the Aboriginal Australians, largely resulting from poor accessibility to heath care resources ${ }^{19}$. In low- and middle-income countries, RHD remains a major public health concern owing to issues of overcrowding, poor hygiene, and low adherence to secondary prophylaxis ${ }^{15}$.

Watkins et al. ${ }^{6}$ estimated that in 2015 there were about 33.4 million people diagnosed with RHD worldwide, with less than $1 \%$ of cases occurring in high-income countries ${ }^{6}$. The number of disability-adjusted life-years due to RHD was 10.5 million, accounting for $0.43 \%$ of global disability-adjusted life-years due to any cause. The highest age-standardized mortality occurred in Oceania, South Asia, and central sub-Saharan Africa, while the highest numbers of deaths due to RHD, in descending order, were observed in India, China, and Pakistan ${ }^{6}$. Latin America demonstrates interesting epidemiological trends for RHD, as it has one of the highest levels of income disparity, with a sizable proportion of the population at high risk ${ }^{20}$. An interesting case in point is Brazil, which has experienced an impressive reduction in RHD incidence and mortality ${ }^{21}$, while its prevalence (1 to 7 cases $/ 1,000)$ remains high compared to developed countries (0.1-0.4 cases/1,000 schoolchildren $)^{21}$.

\section{SUBCLINICAL DISEASE DETECTED BY SCREENING ECHOCARDIOGRAPHY}

During the last decade, interest in early diagnosis for prevention of rheumatic fever recurrence has triggered an increasing number of echocardiography-based screening studies ${ }^{22}$. Furthermore, large-scale screening using portable echocardiographic equipment has become increasingly feasible and extremely effective in high-prevalence regions ${ }^{13,23}$.

Early population-based epidemiologic studies on RHD prevalence were based largely on clinical examination ${ }^{22,23}$. 
The first screening program, including 16 developing countries, relied solely on cardiac auscultation to diagnose RHD $^{24}$. Subsequently, RHD screening began to include echocardiography for confirmation of disease in clinically suspected cases $^{23}$. However, cardiac auscultation has poor sensitivity and specificity for the diagnosis of RHD.

Subsequent studies have consistently highlighted the superiority of echocardiography for detecting subclinical RHD $^{25,26}$, compared to auscultatory screening; hence, auscultatory screening is no longer recommended ${ }^{23}$.

Considering the challenges associated with large-scale screening and the criteria used in diagnosing RHD, the World Heart Federation (WHF) established an evidence-based approach to standardize echocardiographic parameters for the diagnosis of $\mathrm{RHD}^{27}$. These current criteria take into consideration both morphological and functional changes of the left heart valves, and are designed to improve the accuracy of RHD diagnosis in children without a history of ARF. Patients are classified into definite or borderline RHD groups, and further subcategorized within each grouping. There has been a substantial increase in the number of studies on the prevalence of RHD since the establishment of the WHF standardized-echocardiographic criteria screening ${ }^{19,20,25,28-32}$. A recent meta-analysis showed that the prevalence of subclinical RHD is seven to eight times higher than that of clinically manifest disease ${ }^{33}$.

Echocardiographically detected RHD has been termed latent RHD, which includes a broader spectrum of disease, including any RHD identified on echocardiographic screening in the absence of prior ARF or known RHD'. Although echocardiographic screening shows promise for early diagnosis of RHD, the progression of screen-detected disease is not yet fully characterized ${ }^{23}$. Previous studies on the natural history of latent RHD have reported progression of valve lesions in a relatively short follow-up period ${ }^{34}$. However, studies utilizing echocardiography have employed different definitions of progression, and standardization is required in reporting outcomes ${ }^{35}$. Although most children with borderline or mild definite RHD remain stable or demonstrate improvement, they are at substantial risk of progression ${ }^{34}$. Outcomes are best for children with borderline RHD and worst for those with advanced RHD $^{34,36}$. There is also evidence that RHD progression may not be uniform ${ }^{37}$ and the optimal management of latent RHD remains unknown ${ }^{23}$. While early initiation of penicillin prophylaxis will prevent progression to overt $\mathrm{RHD}^{36,38}$, a randomized trial is required to determine the impact of this approach on the outcome of latent RHD.

\section{PATHOGENESIS}

ARF is the result of an autoimmune response to pharyngeal infection with GAS in genetically predisposed individuals, mediated through molecular mimicry ${ }^{5}$. Streptococcal antigens are recognized, processed, and presented by antigen presenting cells such as macrophages and dendritic cells, leading to the production of antibodies by B cells, which cross-react with human components leading to injury in several host sites. The long-term damage to cardiac valves is a more serious complication of ARF.
Typically the first streptococcal throat infection does not trigger an episode of rheumatic fever. One hypothesis is that recurrent infections are able to maintain the germinal center reaction and affinity for antibody maturation, thereby potentiating cross-reactivity ${ }^{39,40}$. As such, preexisting immune complexes would capture more antibodies leading to amplification of the immune response, which further favors the recognition of several host epitopes and propagates tissue damage feeding the disease onset ${ }^{41}$. However, to date, there is still no clear evidence to support the isolated presence of valve-reactive antibodies in the serum of patients with RHD. Moreover, these antibacterial antibodies are often found in patients after uncomplicated streptococcal pharyngitis and also in healthy individuals, supporting the participation of other pathogenic mediators.

Mitral valve damage is initiated by circulating autoantibodies that bind to the endothelial surface of the valves, leading to increased expression of vascular cell adhesion protein 1 (VCAM-1). The activated endothelium facilitates the infiltration of $\mathrm{T}$ lymphocytes into the valvular subendothelium, leading to edema and elongation of the chordae tendineae ${ }^{42,43}$. Intense valvular tissue stretch exposes components of the extracellular matrix, and anti-collagen antibodies are produced in response. These antibodies can deposit in the valve, contributing to a pro-inflammatory milieu. These changes cause the heart valves to be a vulnerable immune-privileged site for degeneration.

It has been proposed that the immune response in RHD may not be merely related to molecular mimicry or failure of the immune system but rather to collagen autoimmunity ${ }^{44}$. The production of autoantibodies against basement-membrane collagen (type IV) on host endothelium serves as the triggering step of pathological processes. In streptococcal infection, $\mathrm{M}$-proteins bind to the $\mathrm{CB} 3$ region of collagen IV, leading to the formation of a complex that promotes conformational changes in collagen structure, thus, initiating an anti-collagen response ${ }^{45-47}$. Thus, a ubiquitous protein can become a self-antigen that contributes to an imbalance between collagen deposition and collagen degradation, culminating in subsequent fibrosis of the valve apparatus in RHD. Mitral valves of patients with RHD demonstrate higher deposition of collagens Type I and Type III, evidence of fibrosis, when compared to non-rheumatic mitral valve controls ${ }^{48}$.

Although some studies suggest that the presence of antibodies is not crucial in triggering RHD pathogenesis, the ability of antibodies to become self-reactive depends on a combination of factors including genetic predisposition, recurrence of infections, and strain virulence. These findings highlight the complexity of the pathogenic mechanisms of RHD.

With respect to cellular immune response, cardiac valves from patients with RHD show an intense inflammatory infiltrate of mononuclear cells. These cells are able to produce cytokines and soluble mediators that affect valvular interstitial cell and valvular endothelial cell functions ${ }^{49,50}$. CD4+ T lymphocytes are highly reactive against cardiac myosin epitopes in RHD $^{51-54}$. Proinflammatory cytokines, such as TNF- $\alpha$, IFN- $\gamma$, IL-1, and IL-17, have been shown to be associated with disease 
progression $^{55,56}$. IL-10, a modulatory cytokine, is present at high levels in patients with RHD and has a direct correlation with the CD8+ T lymphocyte response ${ }^{57-59}$. Macrophages are abundant in valvular inflammatory infiltrate and play an important role in production of both cytokine and matrix metalloproteinases, thus interfering in the remodeling of extracellular matrix components and fibrosis ${ }^{60-62}$.

Calcification is also a very common finding in rheumatic mitral valves. The cellular mechanisms responsible for the calcification in RHD are not well understood. Mineralization occurs in areas of neoangiogenesis, which is associated with inflammation and increased expression of vascular endothelial growth factor (VEGF) ${ }^{63}$. This molecule has the ability to regulate bone remodeling by stimulating osteoblast differentiation ${ }^{64}$. Calcification-competent extracellular vesicles derived from smooth muscle cells, valvular interstitial cells, or macrophages may also be involved in mitral valve mineralization in RHD ${ }^{65-67}$. These cell types are able to produce osteopontin and osteocalcin, demonstrating osteoblast-like processes of calcification such as occur in degenerative calcific aortic stenosis ${ }^{68,69}$. These data suggest that a regulated pro-inflammatory cellular process drives calcification of valve tissue in RHD.

Genetic host predisposition also influences RHD pathogenesis. Susceptibility is heritable, with increased occurrence of ARF in family members and monozygotic twins ${ }^{70,71}$. Polymorphisms in several genes coding for immune-related proteins have been associated with ARF and RHD susceptibility.

In conclusion, the growth in research on RHD pathogenesis has resulted in several advances in current disease understanding ${ }^{72}$. Molecular mimicry is likely essential for induction of autoimmunity and initiation of valve damage during episodes of $\mathrm{ARF}$, and antibodies against collagen could contribute to disease progression, as well as self-reactive clones of Tymphocytes and other cellular components ${ }^{5,72}$. However, the current proposed mechanisms cannot clearly explain the preferential involvement of the mitral valve, or why the disease develops in women in the vast majority of cases. Figure 1 displays a schematic presentation of the mechanisms of RHD pathogenesis.

\section{PATTERNS OF STRUCTURAL VALVULAR ABNORMALITIES OF RHEUMATIC HEART DISEASE}

RHD predominantly affects the mitral valve in essentially all cases, but other valves may also be involved ${ }^{2,12}$. Aortic valve involvement is observed in $20-30 \%$ of cases, and the tricuspid valve may be affected less commonly. The interval between the initial episode of rheumatic fever and clinical evidence of valve disease varies, ranging from a few years to more than 20 years.

Mitral valve regurgitation is the most common valvular lesion in patients with RHD, particularly in the early stages, while pure mitral regurgitation is the most common RHD presentation $^{73-75}$. Rheumatic mitral regurgitation primarily results from morphological changes that reflect chronic scarring of the mitral valve and mitral valve apparatus. Mitral valve stenosis usually occurs many years after the initial episode of $\mathrm{ARF}$, and shares many morphological features with rheumatic

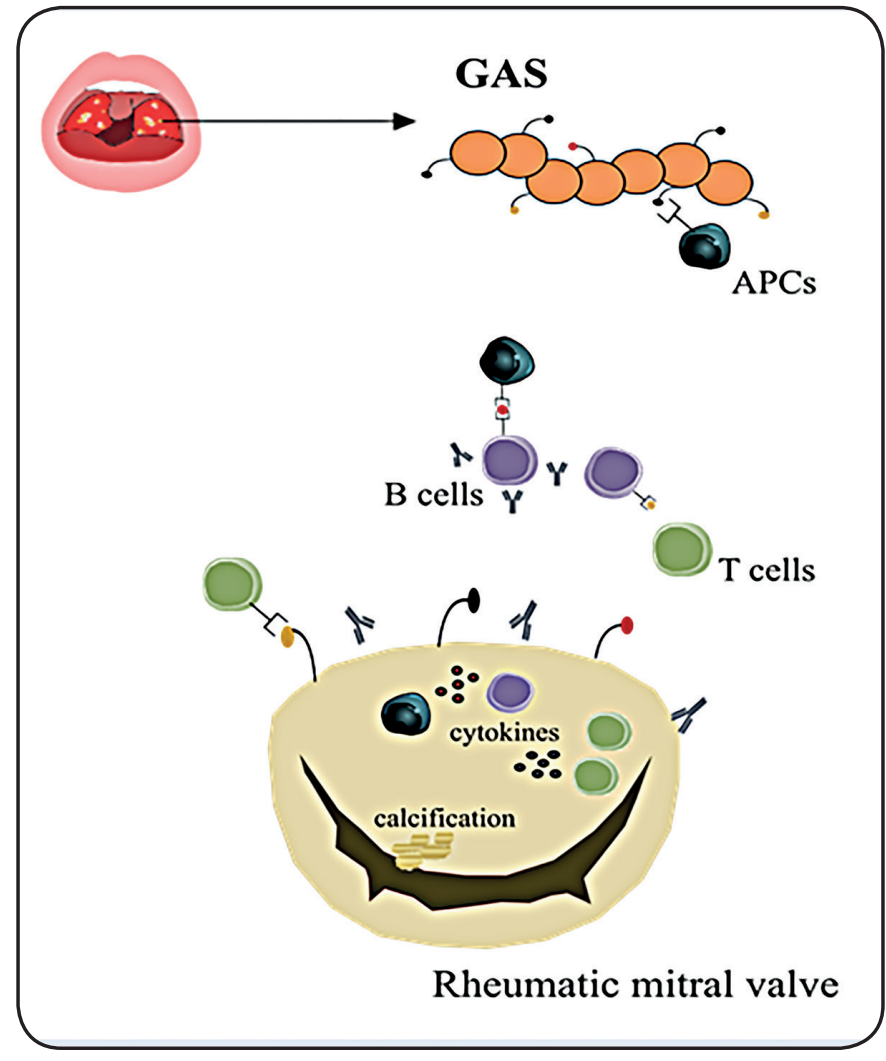

FIGURE 1: Schematic representation of mechanisms of pathogenesis of rheumatic heart disease. Following Group A Streptococcus (GAS) invasion of pharyngeal epithelium, GAS recognize and process bacterial antigens and present them to B lymphocytes. Activated B cells produce antibodies able to recognize epitopes in several sites in host and also activate $T$ lymphocytes. In heart, cross-reactive $\mathrm{T}$ cell clones and antibodies react against heart valves components leading to intense inflammatory process culminating in valve dysfunction

mitral regurgitation ${ }^{2,73}$. The morphological features of mitral stenosis are thickening at the leaflet edges, subvalvular apparatus thickening, shortened chordae tendineae, commissural fusion, calcification, and restricted leaflet motion. Usually, the base and mid-sections of the leaflets move toward the ventricular apex, while the motion of the leaflet tips is restricted owing to fusion of the anterior and posterior leaflets along the medial and lateral commissures $^{27}$. In rheumatic mitral stenosis, two-dimensional echocardiography allows for detailed evaluation of mitral valve morphology, including assessment of leaflet thickness, leaflet mobility, degree of calcification, and extent of subvalvular involvement (Figure 2) ${ }^{75}$.

Although aortic valve disease is less common than mitral valve, aortic valve dysfunction has a more serious effect on left ventricular function, quality of life, and overall prognosis ${ }^{73}$. Rheumatic aortic regurgitation is most commonly seen in combination with rheumatic mitral valve disease (Figure 3) $)^{12}$. Pure aortic valve disease is uncommon. The mechanism for rheumatic aortic regurgitation is most commonly restricted aortic leaflet motion, which occurs as a result of leaflet retraction and thickening ${ }^{73}$. Aortic stenosis is less commonly a result of rheumatic valvular pathology. Data from a large prospective African study reported aortic stenosis in only $9 \%$ of study 


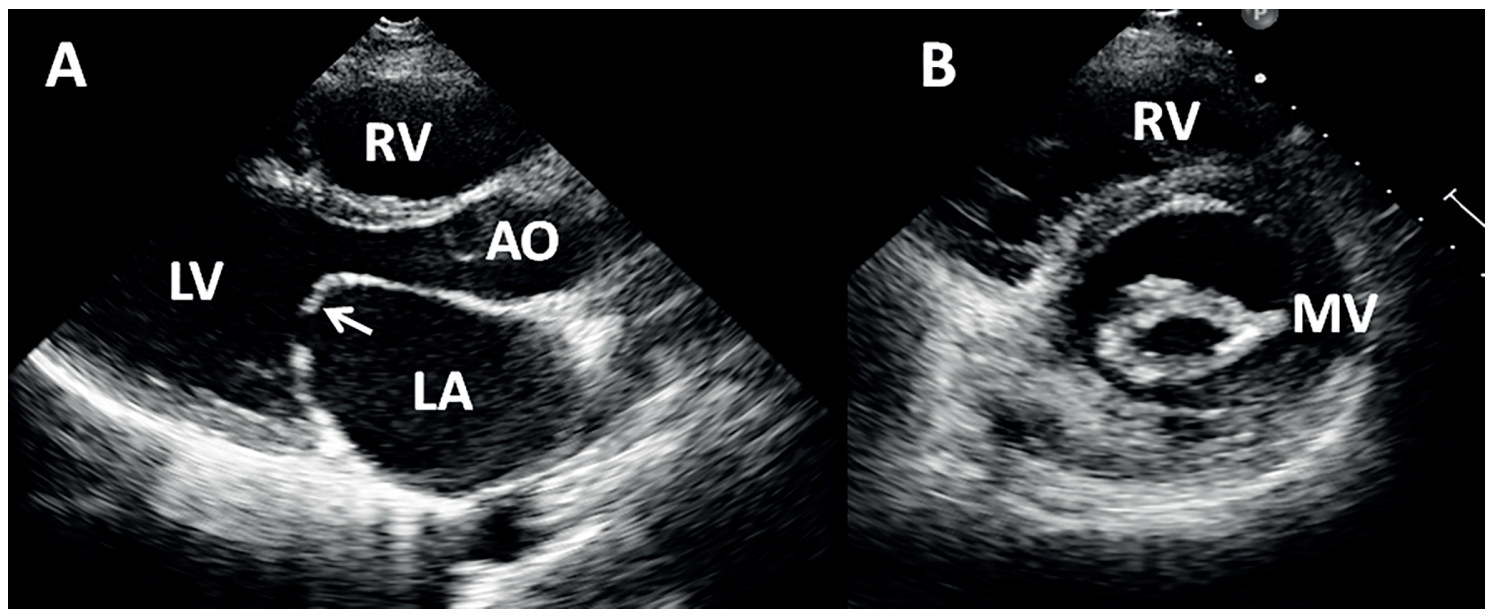

FIGURE 2: Transthoracic echocardiography from patient with severe mitral stenosis in parasternal long-axis (A) and short-axis (B) views. Both leaflets are thickened with pliable anterior leaflet on long-axis view (arrow) and fusion of both commissures on short-axis view. AO: aorta; LA: left atrium; LV: left ventricle; MV: mitral valve; RV: right ventricle.

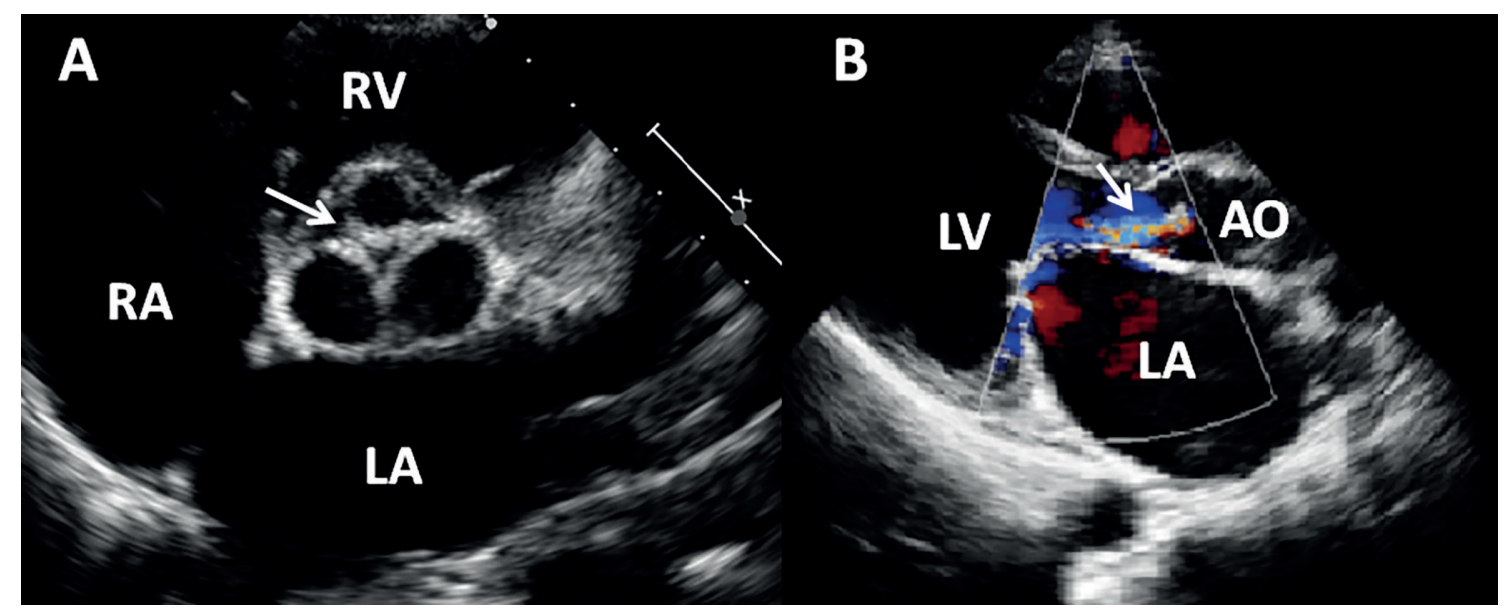

FIGURE 3: Transthoracic echocardiography from 14-year-old child with rheumatic involvement of aortic valve. Parasternal short-axis view (A) showing aortic leaflet thickening (arrow) with mild aortic stenosis associated with pathological aortic regurgitation, seen in parasternal long-axis view by color Doppler (arrow).

AO: aorta; LA: left atrium; LV: left ventricle; RA: right atrium; RV: right ventricle.

subjects $^{12}$. Stenosis occurs secondary to progressive leaflet thickening, commissural fusion, fibrosis, and calcification, usually associated with aortic regurgitation and mitral valve disease.

Rheumatic tricuspid valve involvement is even less common than that of left-sided valves ${ }^{73}$. Regurgitation is a consequence of shortening and retraction of leaflets of the valve as well as shortening and fusion of the chordae tendineae. Tricuspid regurgitation is most often due to tricuspid annulus dilation, in association with mitral valve disease. Rheumatic tricuspid stenosis is rare and occurs in association with mitral stenosis in almost all cases.

\section{4: Preventive strategies}

Preventive strategies for RHD are a set of well-established measures aimed at preventing manifestation of the disease or its complications. These strategies are crucial in the management of patients at risk, and their effectiveness is proven by the low rates of RHD in countries where they were implemented ${ }^{76}$, as well as by the decline in prevalence of the disease worldwide. The World Health Organization (WHO) global action plan targets a $25 \%$ reduction in premature mortality from non-communicable diseases by the year 2025; control and prevention of RHD will play an important role in achieving this goal ${ }^{77}$. Preventive strategies include primordial, primary, secondary, and tertiary prevention, each of which is specific for certain susceptible groups and situations.

Primordial prevention involves prophylactic strategies to avoid streptococcal infection. Improvement in a population's living conditions results in better access to health care and control of the spread of streptococcal pharyngitis, a determinant factor in the pathophysiology of RHD. This type of prevention applies to the general population, and is particularly necessary in socioeconomically disadvantaged contexts. This type of prevention was responsible for the decline in cases of ARF and RHD in most countries in the 20th century even before the 
introduction of antibiotics ${ }^{76}$. The precariousness of this type of prevention in resource-poor settings explains the persistence of rheumatic fever as a major public health challenge among these populations ${ }^{1}$.

Primary prevention of RHD focuses on diagnosis and treatment of streptococcal pharyngitis to decrease the risk of ARF. Once the diagnosis of pharyngitis has been established, treatment within nine days of infection is recommended. This approach prevents the development of rheumatic fever in the majority of susceptible individuals, in addition to avoiding spread of the bacterium between contacts. Intramuscular BPG remains the most widely used antibiotic for GAS pharyngitis ${ }^{78}$. An active school-based sore throat surveillance and treatment adopted in New Zealand demonstrated no statistically significant reduction in the incidence of ARF, along with being unaffordable in the majority of countries with high rates of the disease ${ }^{79}$. Studies are ongoing on the feasibility of a vaccine against $S$. pyogenes ${ }^{80}$. Poor health-seeking literacy and lack of community awareness regarding pharyngitis and RHD are also barriers to primary prevention?

Secondary prevention involves continuous antimicrobial prophylaxis to prevent group A beta-hemolytic streptococcal pharyngitis in individuals previously diagnosed with $\mathrm{ARF}^{78}$. There is strong evidence that secondary prophylaxis reduces the severity of RHD by preventing disease progression ${ }^{1}$. This type of prophylaxis is standardized according to severity of the manifested disease, and takes into account individual risk factors and the epidemiology of the disease in the assessed context. Treatment is prolonged and patient adherence dependent on the patient-physician relationship and satisfactory access to health care. It is essential to emphasize to patients the severity of their condition and the importance of treatment.

The appropriate duration of secondary prophylaxis is controversial, and dependent on several factors, including time elapsed since the last episode of ARF, age, presence of carditis at presentation, and severity of RHD at follow-up ${ }^{78}$. Intramuscular administration of long-acting penicillin preparations every four weeks remains the standard of care in most circumstances $^{78}$. In populations in which the incidence of rheumatic fever is particularly high, including Brazil, the administration of BPG every three weeks is justified and recommended, because serum drug levels may fall below protective levels before the fourth week after administration of this dose of penicillin ${ }^{78}$.

On the basis of these factors, the recommended duration of secondary prophylaxis is usually a minimum of 10 years after the most recent episode of ARF or until age 18-21 years (whichever is longer). Patients with moderate RHD continue prophylaxis until age 30-35, and those with severe RHD continue until age $40^{1,9,81}$.

Secondary prophylaxis remains the most effective type in countries in which primordial and primary preventions are limited. In addition, initiation of penicillin prophylaxis for latent RHD, detected by echocardiography, may prevent advancement to clinically significant disease ${ }^{36,38}$, though this has not yet been proven.

Tertiary prevention aims to prevent complications of established RHD, in order to reduce morbidity and mortality. This entails management of heart failure, control of arrhythmias, adequate monitoring of anticoagulation therapy, prevention of endocarditis, management of complications related to pregnancy, and timely referral for heart surgery ${ }^{9}$. This stage is complex, requiring special resources, specialized professionals, and long-term patient follow-up. Echocardiography has become an essential tool for grading the severity of valvular disease and assessing ventricular function ${ }^{81}$.

The major challenge of this type of prophylaxis is that life-saving valve procedures are not available to the majority of affected RHD patients, contributing to an increased risk of death and other major adverse outcomes ${ }^{12,15,82}$. The REMEDY study (Global Rheumatic Heart Disease Registry) documented high rates of disability and premature death across African and Asian countries, largely attributable to advanced disease at the time of presentation ${ }^{12}$. Strategies to provide high-quality tertiary care for patients with RHD are required, including sustainable cardiac surgical services ${ }^{77}$.

\section{FINAL CONSIDERATIONS}

Although the health-related burden of RHD has declined worldwide, the disease remains a leading cause of death and disability in low- to middle-income countries. Early diagnosis for prevention of rheumatic fever recurrences is an effective strategy to decrease the RHD burden. Echocardiographic screening has been demonstrated to be a powerful tool for early RHD detection, and holds potential for global disease control. Advanced cardiovascular care for patients with late-stage RHD in resourcepoor settings is a challenge. Effective implementation of cardiac surgical services with availability of life-saving valve procedures is essential to manage patients with RHD. Ensuring timely access to definitive surgical care is a key aspect of addressing the current RHD burden, contributing to a decreased risk of death and other major adverse outcomes in patients with advanced disease.

Acknowledgments: We would like to thank the Hospital das Clinicas da UFMG that supports this work.

Conflict of interest: The authors declare that there is no conflict of interest.

Funding support: This study was partly funded by FAPEMIG, CNPq and and MCPN is CNPq fellow.

\section{REFERENCES}

1. Carapetis JR, Beaton A, Cunningham MW, Guilherme L, Karthikeyan G, Mayosi BM, et al. Acute rheumatic fever and rheumatic heart disease. Nat Rev Dis Primers. 2016;2:15084

2. Marijon E, Mirabel M, Celermajer DS, Jouven X. Rheumatic heart disease. Lancet. 2012;379 (9819):953-964.

3. Seckeler MD, Hoke TR. The worldwide epidemiology of acute rheumatic fever and rheumatic heart disease. Clin epidemiol. 2011;3:67-84.

4. Woldu B, Bloomfield GS. Rheumatic heart disease in the twentyfirst century. Curr Cardiol Rep. 2016;18(10):96.

5. Karthikeyan G, Guilherme L. Acute rheumatic fever. Lancet. 2018;392:161-174.

6. Watkins DA, Johnson CO, Colquhoun SM, Karthikeyan G, Beaton A, Bukhman G, et al. Global, regional, and national burden of 
rheumatic heart disease, 1990-2015. N Engl J Med. 2017;377(8): 713-22.

7. Mortality GBD, Causes of Death C. Global, regional, and national age-sex specific all-cause and cause-specific mortality for 240 causes of death, 1990-2013: A systematic analysis for the global burden of disease study 2013. Lancet. 2015;385 (9963):117-71.

8. Global Burden of Disease Study C. Global, regional, and national incidence, prevalence, and years lived with disability for 301 acute and chronic diseases and injuries in 188 countries, 1990-2013: A systematic analysis for the global burden of disease study 2013. Lancet. 2015;386(9995):743-800.

9. Watkins DA, Beaton AZ, Carapetis JR, Karthikeyan G, Mayosi BM, Wyber R, et al. Rheumatic heart disease worldwide: Jacc scientific expert panel. J Am Coll Cardiol. 2018;72(12):1397-1416.

10. Animasahun BA, Madise Wobo AD, Itiola AY, Adekunle MO, Kusimo OY, Thomas FB. The burden of rheumatic heart disease among children in lagos: How are we fairing? Pan Afr Med J. 2018;29:150.

11. Guilherme L, Kalil J. Rheumatic fever and rheumatic heart disease: Cellular mechanisms leading autoimmune reactivity and disease. J Clin Immunol. 2010;30(1):17-23.

12. Zuhlke L, Engel ME, Karthikeyan G, Rangarajan S, Mackie $\mathrm{P}$, Cupido B, et al. Characteristics, complications, and gaps in evidence-based interventions in rheumatic heart disease: The global rheumatic heart disease registry (the remedy study). Eur Heart J. 2015;36(18):1115-22a.

13. Saxena A, Zuhlke L, Wilson N. Echocardiographic screening for rheumatic heart disease: Issues for the cardiology community. Glob Heart. 2013;8(3):197-202.

14. Engelman D, Wheaton GR, Mataika RL, Kado JH, Colquhoun SM, Remenyi B, et al. Screening-detected rheumatic heart disease can progress to severe disease. Heart Asia. 2016;8 (2):67-73.

15. Zuhlke L, Karthikeyan G, Engel ME, Rangarajan S, Mackie P, Cupido-Katya Mauff B, et al. Clinical outcomes in 3343 children and adults with rheumatic heart disease from 14 low- and middleincome countries: Two-year follow-up of the global rheumatic heart disease registry (the remedy study). Circulation. 2016;134(19):145666.

16. Antunes MJ. Challenges in rheumatic valvular disease: Surgical strategies for mitral valve preservation. Glob Cardiol Sci Pract. 2015;2015:9

17. He VY, Condon JR, Ralph AP, Zhao Y, Roberts K, de Dassel JL, et al. Long-term outcomes from acute rheumatic fever and rheumatic heart disease: A data-linkage and survival analysis approach. Circulation. 2016;134(3):222-32.

18. Demirozu ZT, Arat N, Kucukaksu DS. Fine-tuning management of the heart assist 5 left ventricular assist device with two- and threedimensional echocardiography. Cardiovasc J Afr. 2016;27(4):20812 .

19. Roberts K, Maguire G, Brown A, Atkinson D, Remenyi B, Wheaton $G$, et al. Echocardiographic screening for rheumatic heart disease in high and low risk australian children. Circulation. 2014;129(19):1953-61.

20. Nascimento BR, Beaton AZ, Nunes MC, Diamantino AC, Carmo GA, Oliveira KK, et al. Echocardiographic prevalence of rheumatic heart disease in brazilian schoolchildren: Data from the provar study. Int J Cardiol. 2016;219:439-45.

21. Ribeiro AL, Duncan BB, Brant LC, Lotufo PA, Mill JG, Barreto SM. Cardiovascular health in brazil: Trends and perspectives. Circulation. 2016;133(4):422-33.
22. Zuhlke L, Mayosi BM. Echocardiographic screening for subclinical rheumatic heart disease remains a research tool pending studies of impact on prognosis. Curr Cardiol Rep. 2013;15 (3):343.

23. Roberts K, Colquhoun S, Steer A, Remenyi B, Carapetis J. Screening for rheumatic heart disease: Current approaches and controversies. Nat Rev Cardiol. 2013;10(1):49-58.

24. Who programme for the prevention of rheumatic fever/rheumatic heart disease in 16 developing countries: Report from phase i (198690). Who cardiovascular diseases unit and principal investigators. Bull World Health Organ. 1992;70(2):213-18.

25. Beaton A, Okello E, Lwabi P, Mondo C, McCarter R, Sable C. Echocardiography screening for rheumatic heart disease in ugandan schoolchildren. Circulation. 2012;125(25):3127-32.

26. Roberts KV, Brown AD, Maguire GP, Atkinson DN, Carapetis JR. Utility of auscultatory screening for detecting rheumatic heart disease in high-risk children in australia's northern territory. Med J Aust. 2013;199(3):196-9.

27. Remenyi B, Wilson N, Steer A, Ferreira B, Kado J, Kumar K, et al. World heart federation criteria for echocardiographic diagnosis of rheumatic heart disease--an evidence-based guideline. Nat Rev Cardiol. 2012;9(5):297-309.

28. Marijon E, Ou P, Celermajer DS, Ferreira B, Mocumbi AO, Jani D, et al. Prevalence of rheumatic heart disease detected by echocardiographic screening. N Engl J Med. 2007;357:470-6.

29. Marijon E, Celermajer DS, Tafflet M, El-Haou S, Jani DN, Ferreira $\mathrm{B}$, et al. Rheumatic heart disease screening by echocardiography: The inadequacy of world health organization criteria for optimizing the diagnosis of subclinical disease. Circulation. 2009;120(8):663-8.

30. Paar JA, Berrios NM, Rose JD, Caceres M, Pena R, Perez W, et al. Prevalence of rheumatic heart disease in children and young adults in nicaragua. Am J Cardiol. 2010;105:1809-14.

31. Engel ME, Haileamlak A, Zuhlke L, Lemmer CE, Nkepu S, van de Wall M, et al. Prevalence of rheumatic heart disease in 4720 asymptomatic scholars from south africa and ethiopia. Heart. 2015;101:1389-94.

32. Mirabel M, Fauchier T, Bacquelin R, Tafflet M, Germain A, Robillard C, et al. Echocardiography screening to detect rheumatic heart disease: A cohort study of schoolchildren in french pacific islands. Int J Cardiol. 2015;188:89-95.

33. Rothenbuhler M, O'Sullivan CJ, Stortecky S, Stefanini GG, Spitzer E, Estill J, et al. Active surveillance for rheumatic heart disease in endemic regions: A systematic review and meta-analysis of prevalence among children and adolescents. Lancet Glob Health. 2014;2:e717-e726.

34. Beaton A, Aliku T, Dewyer A, Jacobs M, Jiang J, Longenecker CT, et al. Latent rheumatic heart disease: Identifying the children at highest risk of unfavorable outcome. Circulation. 2017;136:2233-44.

35. Karthikeyan G. Measuring and reporting disease progression in subclinical rheumatic heart disease. Heart Asia. 2016;8:74-75.

36. Cannon J, Roberts K, Milne C, Carapetis JR. Rheumatic heart disease severity, progression and outcomes: A multi-state model. J Am Heart Assoc. 2017;6.

37. Zuhlke L, Engel ME, Lemmer CE, van de Wall M, Nkepu S, Meiring A, et al. The natural history of latent rheumatic heart disease in a 5 year follow-up study: A prospective observational study. BMC Cardiovascular Disorders. 2016;16:46.

38. Gewitz MH, Baltimore RS, Tani LY, Sable CA, Shulman ST, Carapetis J, et al. Revision of the jones criteria for the diagnosis of acute rheumatic fever in the era of doppler echocardiography: A scientific statement from the american heart association. Circulation. 2015;131(20):1806-18. 
39. Zhang D, Patel A, Zhu Y, Siegel A, Zalcman SS. Anti-streptococcus igm antibodies induce repetitive stereotyped movements: Cell activation and co-localization with fcalpha/mu receptors in the striatum and motor cortex. Brain Behav Immun. 2012;26 (3):521-33.

40. Gorton D, Sikder S, Williams NL, Chilton L, Rush CM, Govan $\mathrm{BL}$, et al. Repeat exposure to group a streptococcal $\mathrm{m}$ protein exacerbates cardiac damage in a rat model of rheumatic heart disease. Autoimmunity. 2016;49(8):563-70.

41. Dudding BA, Ayoub EM. Persistence of streptococcal group a antibody in patients with rheumatic valvular disease. J Exp Med. 1968;128:1081-98.

42. Roberts S, Kosanke S, Terrence Dunn S, Jankelow D, Duran CM, Cunningham MW. Pathogenic mechanisms in rheumatic carditis: Focus on valvular endothelium. J Infect Dis. 2001;183(3):507-11.

43. Williams RV, Minich LL, Shaddy RE, Veasy LG, Tani LY. Evidence for lack of myocardial injury in children with acute rheumatic carditis. Cardiol Young. 2002;12:519-523.

44. Hudson BG, Tryggvason K, Sundaramoorthy M, Neilson EG. Alport's syndrome, goodpasture's syndrome, and type iv collagen. N Engl J Med. 2003;348 (25):2543-2556.

45. Dinkla K, Talay SR, Morgelin M, Graham RM, Rohde M, NitscheSchmitz DP, et al. Crucial role of the cb3-region of collagen iv in parf-induced acute rheumatic fever. PLoS One. 2009;4 (3):e4666.

46. Dinkla K, Rohde M, Jansen WT, Kaplan EL, Chhatwal GS, Talay SR. Rheumatic fever-associated streptococcus pyogenes isolates aggregate collagen. J Clin Invest. 2003;111:1905-12.

47. Dinkla K, Sastalla I, Godehardt AW, Janze N, Chhatwal GS, Rohde $\mathrm{M}$, et al. Upregulation of capsule enables streptococcus pyogenes to evade immune recognition by antigen-specific antibodies directed to the g-related alpha2-macroglobulin-binding protein grab located on the bacterial surface. Microbes Infect. 2007;9:922-31.

48. Banerjee T, Mukherjee S, Ghosh S, Biswas M, Dutta S, Pattari S, et al. Clinical significance of markers of collagen metabolism in rheumatic mitral valve disease. PLoS One. 2014;9(3):e90527

49. Guilherme L, Cury P, Demarchi LM, Coelho V, Abel L, Lopez AP, et al. Rheumatic heart disease: Proinflammatory cytokines play a role in the progression and maintenance of valvular lesions. Am J Pathol. 2004;165(5):1583-91.

50. Walker GA, Masters KS, Shah DN, Anseth KS, Leinwand LA. Valvular myofibroblast activation by transforming growth factorbeta: Implications for pathological extracellular matrix remodeling in heart valve disease. Circulation Research. 2004;95:253-60.

51. Morris K, Mohan C, Wahi PL, Anand IS, Ganguly NK. Increase in activated $t$ cells and reduction in suppressor/cytotoxic $t$ cells in acute rheumatic fever and active rheumatic heart disease: A longitudinal study. J Infect Dis. 1993;167(4):979-83.

52. Guilherme L, Cunha-Neto E, Coelho V, Snitcowsky R, Pomerantzeff $\mathrm{PM}$, Assis RV, et al. Human heart-infiltrating t-cell clones from rheumatic heart disease patients recognize both streptococcal and cardiac proteins. Circulation. 1995;92:415-20.

53. Bhatnagar A, Grover A, Ganguly NK. Superantigen-induced t cell responses in acute rheumatic fever and chronic rheumatic heart disease patients. Clin Exp Immunol. 1999;116(1):100-6.

54. Fae KC, da Silva DD, Oshiro SE, Tanaka AC, Pomerantzeff PM, Douay $\mathrm{C}$, et al. Mimicry in recognition of cardiac myosin peptides by heart-intralesional $\mathrm{t}$ cell clones from rheumatic heart disease. Journal of immunology. 2006;176:5662-5670.

55. Toor D, Vohra H. Immune responsiveness during disease progression from acute rheumatic fever to chronic rheumatic heart disease. Microbes and infection. 2012;14:1111-7.
56. Ellis NM, Li Y, Hildebrand W, Fischetti VA, Cunningham MW. T cell mimicry and epitope specificity of cross-reactive $t$ cell clones from rheumatic heart disease. J Immunol. 2005 15;175(8):5448-56.

57. Rowbottom AW, Lepper MA, Garland RJ, Cox CV, Corley EG. Interleukin-10-induced cd8 cell proliferation. Immunology. 1999;98 (1):80-9.

58. MacNeil IA, Suda T, Moore KW, Mosmann TR, Zlotnik A. Il10, a novel growth cofactor for mature and immature $\mathrm{t}$ cells. J Immunol. 1990;145(12):4167-73.

59. Sharma N, Toor D. Interleukin-10: Role in increasing susceptibility and pathogenesis of rheumatic fever/rheumatic heart disease. Cytokine. 2017;90:169-76.

60. LaRock $\mathrm{CN}$, Nizet V. Inflammasome/il-1beta responses to streptococcal pathogens. Front Immunol. 2015;6:518.

61. Yi YS. Role of inflammasomes in inflammatory autoimmune rheumatic diseases. Korean J Physiol Pharmacol. 2018;22(1):1-15.

62. Gasse P, Riteau N, Vacher R, Michel ML, Fautrel A, di Padova F, et al. Il-1 and il-23 mediate early il-17a production in pulmonary inflammation leading to late fibrosis. PloS one. 2011;6 (8):e23185.

63. Rajamannan NM, Nealis TB, Subramaniam M, Pandya S, Stock $\mathrm{SR}$, Ignatiev CI, et al. Calcified rheumatic valve neoangiogenesis is associated with vascular endothelial growth factor expression and osteoblast-like bone formation. Circulation. 2005;111:3296-301.

64. Deckers MM, van Bezooijen RL, van der Horst G, Hoogendam J, van Der Bent C, Papapoulos SE, et al. Bone morphogenetic proteins stimulate angiogenesis through osteoblast-derived vascular endothelial growth factor a. Endocrinology. 2002;143 (4):1545-53.

65. Hutcheson JD, Goettsch C, Bertazzo S, Maldonado N, Ruiz JL, Goh W, et al. Genesis and growth of extracellular-vesicle-derived microcalcification in atherosclerotic plaques. Nat Mater. 2016;15 (3):335-43.

66. New SE, Goettsch C, Aikawa M, Marchini JF, Shibasaki M, Yabusaki $\mathrm{K}$, et al. Macrophage-derived matrix vesicles: An alternative novel mechanism for microcalcification in atherosclerotic plaques. Circ Res. 2013;113(1):72-7.

67. Krohn JB, Hutcheson JD, Martinez-Martinez E, Aikawa E. Extracellular vesicles in cardiovascular calcification: Expanding current paradigms. J Physiol. 2016;594:2895-2903.

68. Rajamannan NM, Evans FJ, Aikawa E, Grande-Allen KJ, Demer LL, Heistad DD, et al. Calcific aortic valve disease: Not simply a degenerative process: A review and agenda for research from the national heart and lung and blood institute aortic stenosis working group. Executive summary: Calcific aortic valve disease-2011 update. Circulation. 2011;124(16):1783-91.

69. Yutzey KE, Demer LL, Body SC, Huggins GS, Towler DA, Giachelli $\mathrm{CM}$, et al. Calcific aortic valve disease: A consensus summary from the alliance of investigators on calcific aortic valve disease. Arterioscler Thromb Vasc Biol. 2014;34(11):2387-93.

70. Guilherme L, Ramasawmy R, Kalil J. Rheumatic fever and rheumatic heart disease: Genetics and pathogenesis. Scand J Immunol. 2007;66(2-3):199-207.

71. Bryant PA, Robins-Browne R, Carapetis JR, Curtis N. Some of the people, some of the time: Susceptibility to acute rheumatic fever. Circulation. 2009;119(5):742-53.

72. Tandon R, Sharma M, Chandrashekhar Y, Kotb M, Yacoub MH, Narula J. Revisiting the pathogenesis of rheumatic fever and carditis. Nat Rev Cardiol. 2013 ;10(3):171-7.

73. Remenyi B, ElGuindy A, Smith SC, Jr., Yacoub M, Holmes DR, Jr. Valvular aspects of rheumatic heart disease. Lancet. 2016;387 (10025):1335-46. 
74. Rowe JC, Bland EF, Sprague HB, White PD. The course of mitral stenosis without surgery: Ten- and twenty-year perspectives. Ann Inern Med. 1960;52:741-49.

75. Saxena A, Desai A, Narvencar K, Ramakrishnan S, Thangjam RS, Kulkarni S, et al. Echocardiographic prevalence of rheumatic heart disease in indian school children using world heart federation criteria - a multi site extension of rheumatic study (the e-rheumatic study). Int J Cardiol 2017;249:438-42.

76. Gordis L. The virtual disappearance of rheumatic fever in the united states: Lessons in the rise and fall of disease. T. Duckett jones memorial lecture. Circulation. 1985;72(6):1155-62.

77. White A. Who resolution on Rheumatic Heart Disease. European Heart Journal. 2018;39(48):4233.

78. Gerber MA, Baltimore RS, Eaton CB, Gewitz M, Rowley AH, Shulman ST, et al. Prevention of rheumatic fever and diagnosis and treatment of acute streptococcal pharyngitis: A scientific statement from the american heart association rheumatic fever, endocarditis, and kawasaki disease committee of the council on cardiovascular disease in the young, the interdisciplinary council on functional genomics and translational biology, and the interdisciplinary council on quality of care and outcomes research: Endorsed by the american academy of pediatrics. Circulation. 2009;119(11):1541-51.

79. Lennon D, Stewart J, Farrell E, Palmer A, Mason H. School-based prevention of acute rheumatic fever: A group randomized trial in new zealand. Pediatr Infect Dis J. 2009;28(9):787-94.

80. Steer AC, Carapetis JR, Dale JB, Fraser JD, Good MF, Guilherme L, et al. Status of research and development of vaccines for streptococcus pyogenes. Vaccine. 2016;34(26):2953-8.

81. Nishimura RA, Otto CM, Bonow RO, Carabello BA, Erwin JP, 3rd, Guyton RA, et al. 2014 aha/acc guideline for the management of patients with valvular heart disease: A report of the american college of cardiology/american heart association task force on practice guidelines. J Thorac Cardiovasc Surg. 2014;148(1):e1-e132.

82. Zilla P, Bolman RM, Yacoub MH, Beyersdorf F, Sliwa K, Zuhlke L, et al. The cape town declaration on access to cardiac surgery in the developing world. Cardiov J Africa. 2018;29 (4):256-9. 